\title{
Incidental retroaortic innominate vein in a patient with acute aortic dissection
}

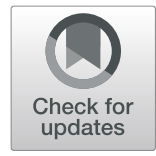

Hideki Sasaki ${ }^{*}$ (D), Takashi Harada', Hiroshi Ishitoya ${ }^{1}$ and Osamu Sasaki ${ }^{2}$

\begin{abstract} protection.

Keywords: Retroaortic innominate vein, Acute aortic dissection

\section{Background}

Retroaortic innominate vein is a rare anomaly. It has been reported in patients with congenital anomalies such as Tetralogy of Fallot or right aortic arch. However, an isolated retroaortic innominate vein is quite rare. We present a patient with a diagnosis of Stanford type A acute aortic dissection in whom a retroaortic innominate vein was incidentally found on computed tomography.
\end{abstract}

Background: Retroaortic innominate vein is a rare anomaly. It has been reported in patients with congenital anomalies such as Tetralogy of Fallot or right aortic arch. However, isolated retroaortic innominate vein is quite rare.

Case presentation: A 63-year-old man was transferred to our institution because of Stanford type A acute aortic dissection. Incidentally, we noticed that the left innominate vein coursed under the aortic arch and was directed into the superior vena cava on computed tomography. We performed emergent hemiarch replacement.

Conclusions: Attention must be paid to the cannulation site for venous uptake and the method of myocardial

\section{Case presentation}

A 63-year-old man was transferred to our institution because of Stanford type A acute aortic dissection (AAAD). Incidentally, we noticed that the left innominate vein coursed under the aortic arch and was directed into the superior vena cava (SVC) on contrast-enhanced computed tomography (CT) (Fig. 1). We decided to perform emergent surgery. Median sternotomy approach was used. After the pericardium was opened, we confirmed that the left innominate vein coursed beneath the aortic arch. We established cardiopulmonary bypass

\footnotetext{
* Correspondence: baggio199869@gmail.com

'Department of Cardiovascular Surgery, Ehime Prefectural Central Hospital, 83 Kasuga-cho, Matsuyama, Ehime 790-0024, Japan

Full list of author information is available at the end of the article
}

(CPB) using right subclavian and left femoral artery inflow with right atrial (RA) drainage. Both antegrade cardioplegia and retrograde cardioplegia were used for myocardial protection. A retrograde cardioplegic cannula was inserted into the coronary sinus under transesophageal echo (TEE) guidance. Hemiarch replacement was performed with hypothermic circulatory arrest (bladder temperature $25^{\circ} \mathrm{C}$ ). An intimal tear was found in the small curvature of the proximal aortic arch, which was excluded. Antegrade selective cerebral perfusion was commenced for brain protection. Circulatory arrest time was $51 \mathrm{~min}$. Aortic cross clamp time was $117 \mathrm{~min}$, with CPB time of $211 \mathrm{~min}$. The subsequent postoperative course was uneventful. The patient was discharged from our institution on the 18th postoperative day. We have followed him in the outpatient clinic.

\section{Discussion}

The left innominate vein is usually located anterosuperior to the aortic arch, and connects with the right innominate vein, forming the SVC. However, it rarely courses via an alternative pathway. Kerschner reported the first case of an alternative course of the left innominate vein more than one hundred years ago [1]. This rare 


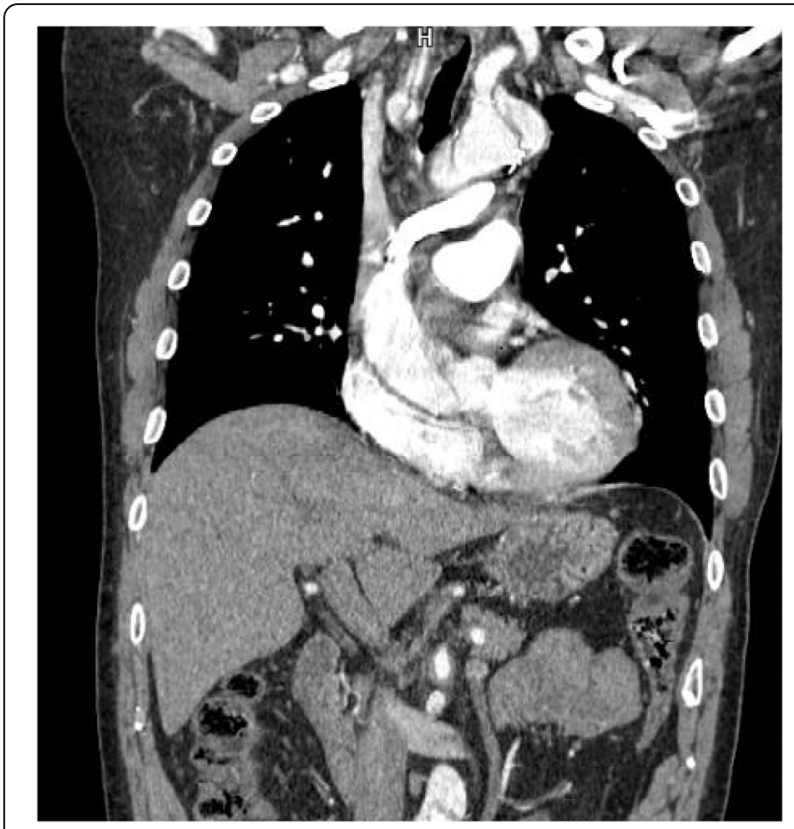

Fig. 1 Left innominate vein courses underneath aortic arch and is directed into superior vena cava

anomaly has been reported in patients with cardiac anomalies. Its incidence is $0.55 \%$ among patients with congenital heart anomaly. It is commonly related to tetralogy of Fallot or right aortic arch [2]. Cases of an isolated retroaortic innominate vein (RAIV) are extremely rare. Nagashima et al. reported that its incidence was $0.02 \%$ ( 1 of 4805 ) in patients without congenital heart anomaly [3]. During normal fetal development, systemic veins develop from paired anterior and posterior cardinal veins. They unite on each side, and form common cardinal veins. Venous return eventually directs into the sinus venosus. The anterior cardinal veins continue to the bifurcation of the internal jugular veins and subclavian veins on each side. However, a large part of the left anterior cardinal vein vanishes. Venous flow from the left side of the head and arm directs into two anastomotic plexuses (superior and inferior channels), and eventually reaches the right anterior cardinal vein by the eighth week [2]. Usually, the inferior transverse capillary plexus regresses, and the superior transverse capillary plexus becomes the left innominate vein (Fig. 2a). The left common cardinal vein becomes the coronary sinus. The oblique vein of Marshall is formed by the left anterior cardinal vein [4]. It is not clear how the RAIV is formed. A possible explanation is failure of usual development of the superior transverse anastomosis and, as a result, an alternative pathway in the caudal position remains and forms the RAIV [4].

RAIV can be problematic if contrast CT is not performed in clinical settings. It may be difficult to insert a central venous catheter from the left jugular/subclavian vein. Permanent pacemaker lead insertion from the left subclavian vein can be hazardous [5]. When surgery is performed in patients with AAAD using retrograde cardioplegia, insertion of the cannula into the coronary sinus under direct vision can be a problem. If insertion of the cannula without opening the RA under TEE guidance is used, RAIV does not cause a problem. If insertion of the cannula into the coronary sinus under direct vision with bicaval drainage is chosen, the site of SVC cannulation should be placed more caudally. Moreover, the SVC must be snared more caudally (Fig. 2b). Otherwise, blood from the RAIV returns into the RA, which makes it difficult to insert a retrograde cardioplegic cannula into the coronary sinus. Furthermore, attention
A

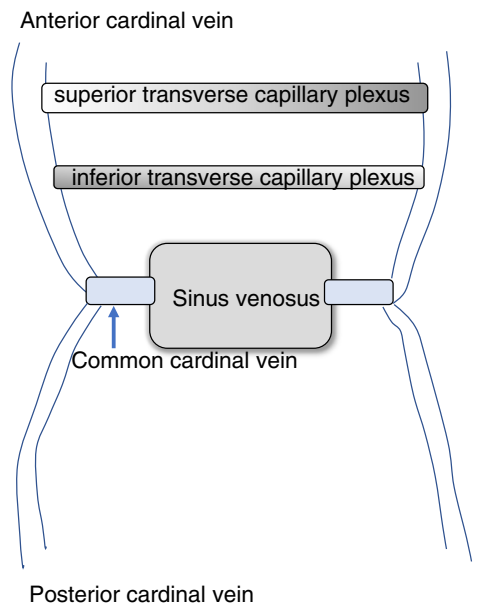

B

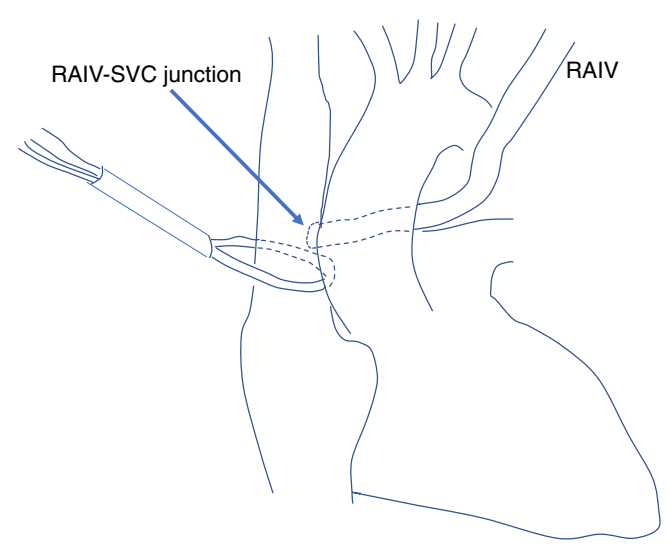

Fig. 2 a. Schema of embryology. $\mathbf{b}$ If insertion of the cannula into the coronary sinus under direct vision with bicaval drainage is chosen, the SVC must be snared more caudally 
should paid to avoiding injury of the RAIV when we encircling the SVC for snaring.

Although we did not perform total arch replacement (TAR) in this patient, it is required when an intimal tear is located in the aortic arch. RAIV may prevent surgeons from looking at the distal aortic arch and performing maneuvers for anastomosis. The higher possibility of injury to the RAIV should be considered while dissecting the posterior part of the aorta and aortic arch. When surgeons retract the RAIV to acquire a good operative field, laceration and subsequent bleeding may occur. When bleeding occurs from the junction between the RAIV and SVC, it might be difficult to control it. Furthermore, strong retraction for a long time can cause thrombosis in the RAIV, which might lead to catastrophic pulmonary embolism. If aortic surgery is required in patents with RAIV via left thoracotomy, the same attention is mandatory for proximal anastomosis.

\section{Conclusion}

Attention must be paid to the cannulation site for venous uptake and the method of myocardial protection when surgery is performed in patients with acute aortic dissection and RAIV.

\section{Supplementary information}

Supplementary information accompanies this paper at https://doi.org/10. 1186/s13019-020-01318-5.

\section{Additional file 1 .}

\section{Abbreviations}

AAAD: Stanford type A acute aortic dissection; SVC: Superior vena cava; CT: Computed tomography; CPB: Cardiopulmonary bypass; RA: Right atrium; TEE: Transesophageal echo; RAIV: Retroaortic innominate vein; TAR: Total arch replacement

\section{Acknowledgements}

Not applicable.

\section{Authors' contributions}

Study conception: HS, Data collection: TH, Analysis: OS, Investigation: $\mathrm{TH}, \mathrm{HI}$, Writing: HS, Critical review and revision: all authors, Final approval of the article: all authors, Accountability for all aspects of the work: all authors.

\section{Funding}

Not applicable.

\section{Availability of data and materials}

Not applicable.

\section{Ethics approval and consent to participate}

The Institutional Review board of Ehime Prefectural Central Hospital approved this study on May 15th, 2020 (IRB number; ken 02-17).

\section{Consent for publication}

Written informed consent was obtained from the patient for publication of this case report.

\section{Competing interests}

The authors declare that they have no competing interests.

\section{Author details}

1Department of Cardiovascular Surgery, Ehime Prefectural Central Hospital, 83 Kasuga-cho, Matsuyama, Ehime 790-0024, Japan. ${ }^{2}$ Division of Internal Medicine, Tokyo-Shinagawa Hospital, 6-3-22, Higashi-Oi, Shinagawa, Tokyo 140-8522, Japan.

Received: 27 July 2020 Accepted: 21 September 2020

Published online: 29 September 2020

\section{References}

1. Kerschner L. Morphologie der vena cava inferior. Anat Anz. 1888;3:808-23.

2. Kulkarni $\mathrm{S}$, Jain $\mathrm{S}$, Kasar P, Garekar S, et al. Retroaortic left innominate vein incidence, association with congenital heart defects, embryology, and clinical significance. Ann Pediatr Cardiol. 2008;1(2):139-41. https://doi.org/10. 4103/0974-2069.43881.

3. Nagashima M, Shikata F, Okamura T, et al. Anomalous subaortic left brachiocephalic vein in surgical cases and literature review. Clin Anat. 2010; 23:9505.

4. Gerlis LM, Ho SY. Anomalous subaortic position of the brachiocephalic (innominate) vein: a review of published reports and report of three new cases. Br Heart J. 1989;61 (6):540-5 Review.

5. Semionov A, Kosiuk J. Incidental retroaortic left innominate vein in adult patient. Radiol Case Rep. 2017;12(3):475-8. https://doi.org/10.1016/j.radcr. 2017.05.005.

\section{Publisher's Note}

Springer Nature remains neutral with regard to jurisdictional claims in published maps and institutional affiliations. 